\title{
Effect of platform switching size and vertical implant depth on crestal bone remodeling: a histological animal study
}

\author{
Gehrke, Sergio A. 1; Pérez, Leticia 2; Mazon, Patricia 3; De Aza, \\ Piedad N. 4 \\ 1 Biotecnos - Technology and Science, Montevideo, Uruguay; ${ }^{2}$ Laboratorio de Interacciones \\ Moleculares, UDELAR, Montevideo, Uruguay; ${ }^{3}$ Departamento de Materiales, Óptica y Tecnologia \\ Electrónica, Universidad Miguel Hernández, Elche (Alicante), Spain; 4 Instituto de Bioingenieria. \\ Universidad Miguel Hernández, Elche, (Alicante), Spain.
}

\section{Abstract}

Background: Functional and dimensional maintenance of the soft tissue seal in dental implants can determinate better long-term condition, and an adequate amount of bone around the implant reduces the risk of the abutment loosening from the implant. Aim: The present animal study aimed to analyze the possible relationship between the combination of different platform switching and vertical implant depth and remodeling of the crestal bone. Material and Methods: Sixty conical Morse taper dental implants were placed in the tibia of 15 rabbits, hence 2 implants per tibia. Three different implant diameters were used, 3.5$\mathrm{mm}, 4.0-\mathrm{mm}$ and $4.5-\mathrm{mm}$ ( $\mathrm{n}=3 \times 20$ implants). The implants were placed either $1-\mathrm{mm}$ or $2.0-\mathrm{mm}$ subcortically, based on a randomized allocation. A transmucosal healing abutment with a diameter of $3.3 \mathrm{~mm}$ and height of $3.5 \mathrm{~mm}$ was secured to the implants. Eight weeks after the implantation surgery, histological samples were prepared and analyzed quantitatively. The extent of new bone formation was appraised by two measurements. l.e., the relative area of new bone formed between the osteotomy wall and the vertical surface of the abutment (\%), and the height from the implant shoulder to the most cervical crest of the new bone $(\mathrm{mm})$. Parametric and nonparametric statistical tests were applied to assess the significance of observed group differences ( $n+6 \times 10$ implants). Results; New bone formation between the osteotomy wall and the vertical surface of the abutment cervically to the implant shoulder was observed in all 6 groups. The highest and lowest new bone formation measured by the bone height was observed respectively for the $3.5 \mathrm{~mm}$ implants placed $2 \mathrm{~mm}$ subcrestally $(1.2 \mathrm{~mm}$ ) versus the 4.0 and $4.5 \mathrm{~mm}$ implants placed $1 \mathrm{~mm}$ subcrestally $(0.6 \mathrm{~mm})(\mathrm{p} 120 \%)$ and the 4 and $4.5 \mathrm{~mm}(\sim 55 \%)$ implants. Conclusions: In this animal model, both the implant diameter and vertical placement level influence the crestal bone behavior during the healing process.

\section{Methods and Materials}

\section{Implants installation and parameters analyzed:}
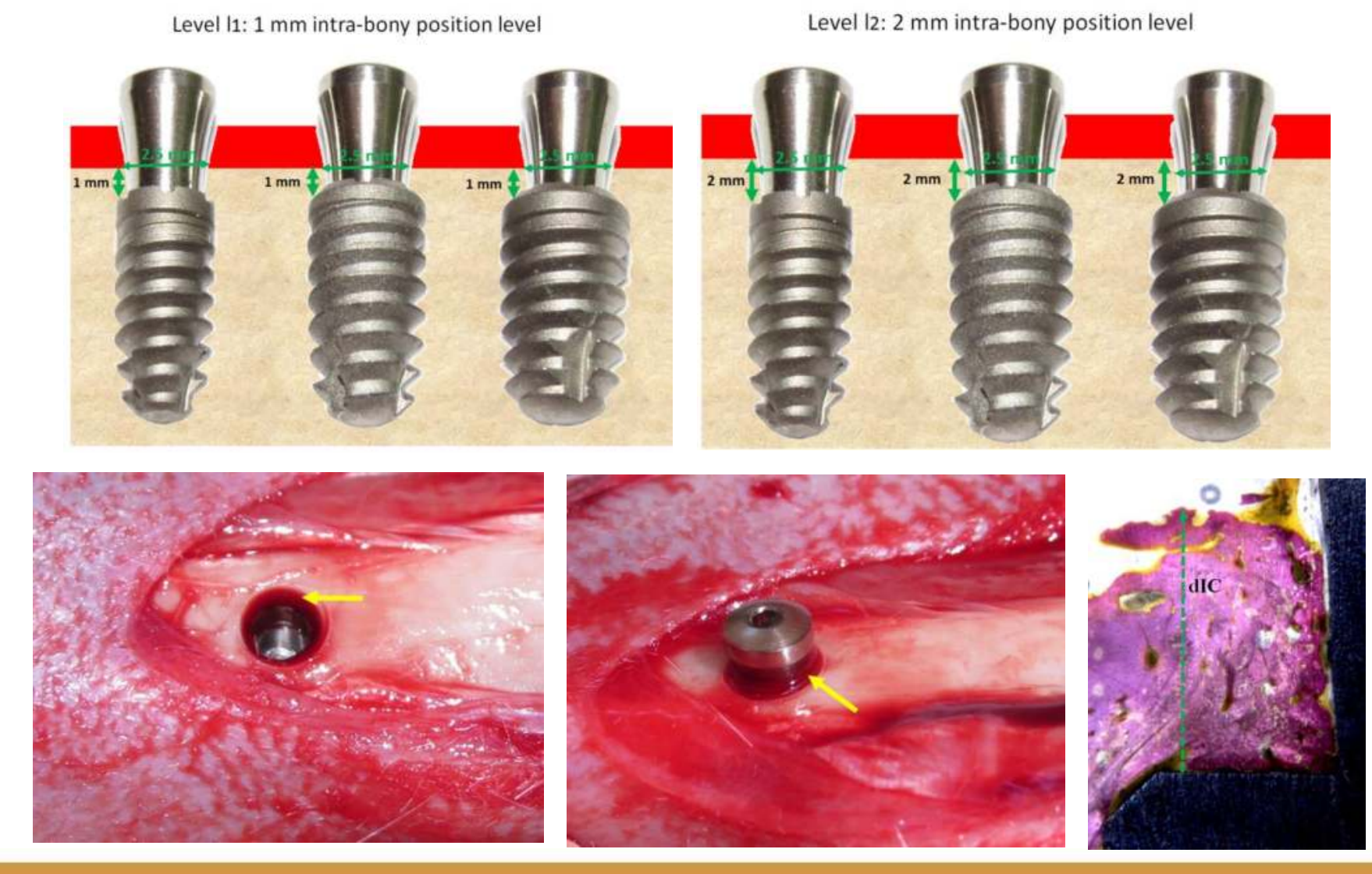

\section{Background and Aim}

Functional and dimensional maintenance of the soft tissue seal in dental implants can determinate better long-term condition, and an adequate amount of bone around the implant reduces the risk of the abutment loosening from the implant. The loss of a tooth initiates a progressive loss of the horizontal and vertical dimensions of the alveolar bone, mainly during the first 90 days. Histologically was showed that a minimum bone loss and minimum inflammatory infiltration occurred when the microgap between the implant platform and the abutment was transferred coronally away from the alveolar bone crest. Supposedly, by using the implant with a Morse taper internal connection, this problem could be decrease or resolved. However, in recent review, the authors concluded that currently, there are no implant connection design 2 or more pieces that can provide a complete seal, which prompts bacterial leakage. This study concluded that microbial leakage may not be of clinical importance. However, reducing of leakage to physiological and tolerable levels in implants with a Morse taper internal connection can be considered as beneficial from clinical perpective. The purpose of the present experimental study was to investigate the behavior of surrounding crestal bone around Morse taper implants as a function of to implant diameter and vertical placement depth relative to the cortical bone level.

\section{Methods and Materials}

Sixty conical Morse taper dental implants were placed in the tibia of 15 rabbits, hence 2 implants per tibia. Three different implant diameters were used, $3.5-\mathrm{mm}, 4.0-\mathrm{mm}$ and $4.5-\mathrm{mm}$ ( $\mathrm{n}=3 \times 20$ implants). The implants were placed either $1-\mathrm{mm}$ or $2.0-\mathrm{mm}$ subcortically, based on a randomized allocation. A transmucosal healing abutment with a diameter of $3.3 \mathrm{~mm}$ and height of $3.5 \mathrm{~mm}$ was secured to the implants. Eight weeks after the implantation surgery, histological samples were prepared and analyzed quantitatively. The extent of new bone formation was appraised by two measurements. I.e., the relative area of new bone formed between the osteotomy wall and the vertical surface of the abutment (\%), and the height from the implant shoulder to the most cervical crest of the new bone $(\mathrm{mm})$.

\section{Results}

Histomorphological analysis and measurements:

Rsults of the implantations at $1 \mathrm{~mm}$ intra-bone:
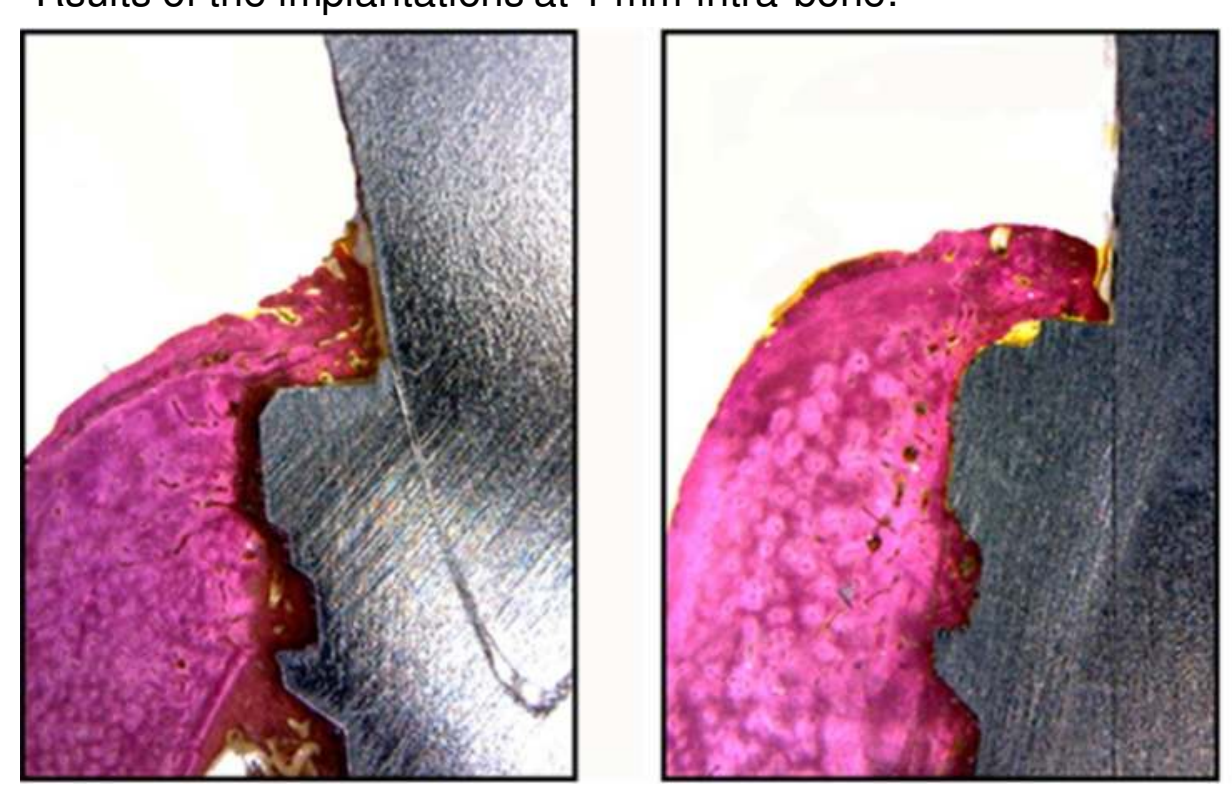

Rsults of the implantations at $2 \mathrm{~mm}$ intra-bone:

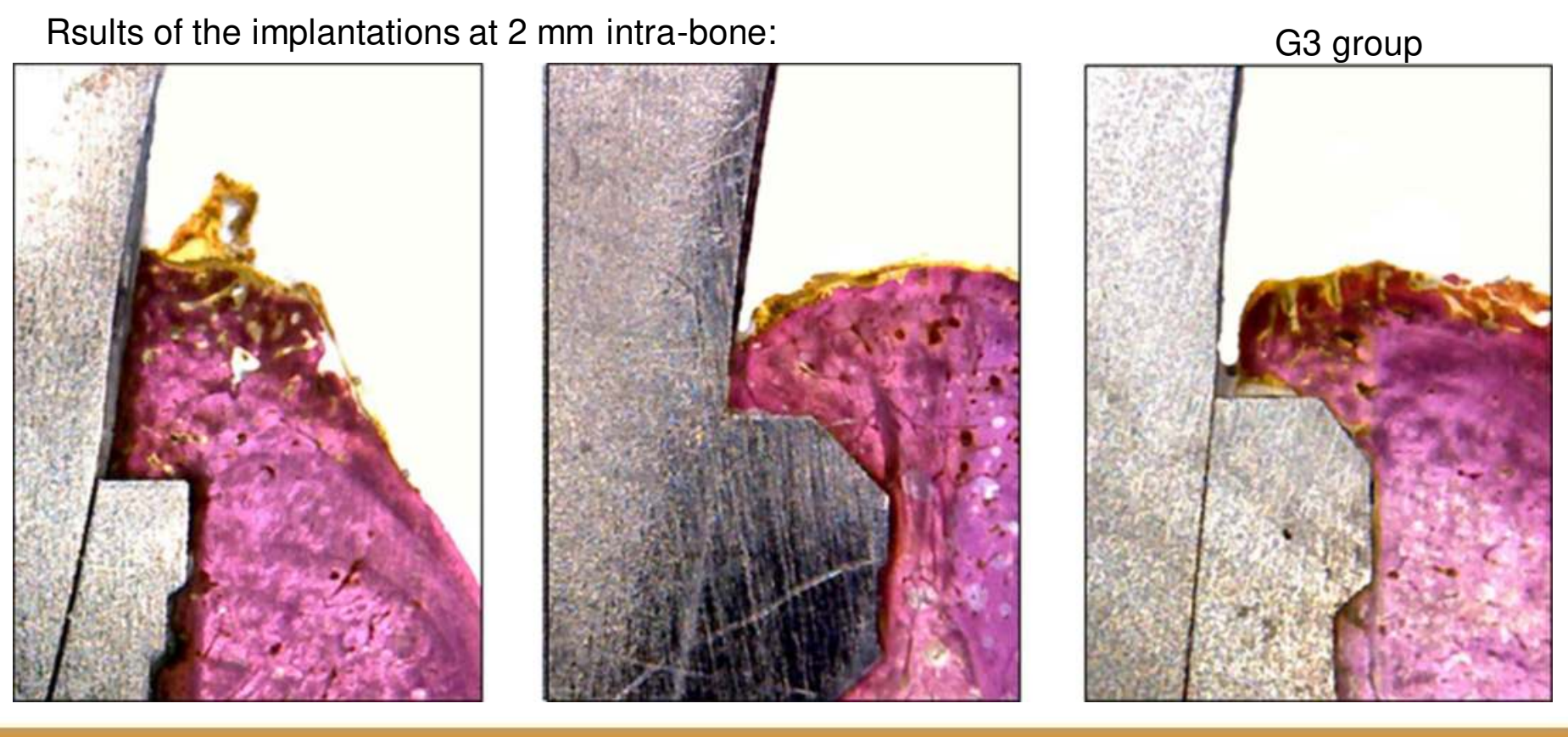

Conclusion

Within the limitations of this study, the histological analysis at samples removed 8-weeks after the implantations showed that the implant diameter and vertical placement depth are important factors affecting the crestal bone formation and tissue seal around of the abutments. 